\title{
Erratum to: Regional brain dysregulation of Ca2+-handling systems in ketamine-induced rat model of experimental psychosis
}

\author{
Malwina Lisek $^{1}$ - Tomasz Boczek ${ }^{1} \cdot$ Bozena Ferenc $^{1} \cdot$ Ludmila Zylinska $^{1}$
}

Published online: 4 February 2016

(C) Springer-Verlag Berlin Heidelberg 2016

Erratum to: Cell Tissue Res (2015)

DOI 10.1007/s00441-015-2332-3

In the original publication, the below funding information was not included.

This work was supported by the Polish National Science Centre Grant based on decision UMO-2012/05/D/NZ4/02982 and, in part, by Medical University of Lodz grants no. 503/ 6-086-02/503-01 and 502-03/6-086-02/502-64-061.

The online version of the original article can be found at http://dx.doi.org/ $10.1007 / \mathrm{s} 00441-015-2332-3$.

Malwina Lisek

malwina.lisek@umed.lodz.pl

1 Department of Molecular Neurochemistry, Faculty of Health Sciences, Medical University of Lodz, 6/8 Mazowiecka Street, 92215 Lodz, Poland 